\title{
PV-Tiles: Towards Closely-Coupled Photovoltaic and Digital Materials for Useful, Beautiful and Sustainable Interactive Surfaces
}

\author{
Yogesh Kumar Meena, ${ }^{1}$ Krishna Seunarine, ${ }^{1}$ Deepak Ranjan Sahoo, ${ }^{1}$ \\ Simon Robinson, ${ }^{1}$ Jennifer Pearson, ${ }^{1}$ Chi Zhang, ${ }^{1}$ Matt Carnie, ${ }^{2}$ Adam Pockett, ${ }^{2}$ \\ Andrew Prescott, ${ }^{2}$ Suzanne Thomas, ${ }^{2}$ Harrison Ka Hin Lee, ${ }^{2}$ Matt Jones ${ }^{1}$
}

\begin{abstract}
${ }^{1}$ Computational Foundry, Swansea University, UK
$\{$ y.k.meena, krishna.seunarine, d.r.sahoo, s.n.w.robinson

chi.zhang, j.pearson, matt.jones \} @swansea.ac.uk

${ }^{2}$ Engineering, Swansea University, UK

$\{$ m.j.carnie, adam.pockett, a.prescott,

s.k.thomas, k.h.lee \} @ swansea.ac.uk
\end{abstract}

\begin{abstract}
The interactive, digital future with its seductive vision of Internet-of-Things connected sensors, actuators and displays comes at a high cost in terms of both energy demands and the clutter it brings to the physical world. But what if such devices were made of materials that enabled them to self-power their interactive features? And, what if those materials were directly used to build aesthetically pleasing environments and objects that met practical physical needs as well as digital ones? In this paper we introduce PV-Tiles - a novel material that closely couples photovoltaic energy harvesting and light sensing materials with digital interface components. We consider potential contexts, use-cases and light gestures surfaced through co-creation workshops; and, present initial technological designs and prototypes. The work opens a new set of opportunities and collaborations between HCI and material science, stimulating technical and design pointers to accommodate and exploit the material's properties.
\end{abstract}

\section{Author Keywords}

Self-powered devices, Internet of Things, sustainability, connected home, interaction design.

\section{CCS Concepts}

-Human-centered computing $\rightarrow$ User studies; Gestural input; Touch screens;

\section{INTRODUCTION}

William Morris, founder of the British Arts and Crafts movement, laid down this 'golden rule' for home owners: "Have nothing in your houses that you do not know to be useful, or believe to be beautiful" [22]. In his own work he achieved the dual of both beauty and utility. One hundred and twenty years since Morris' death, in the light of the climate change crises, those working on materials and objects for home use might

(C) Yogesh Kumar Meena, Krishna Seunarine, Deepak Ranjan Sahoo, Simon Robinson, Jennifer Pearson, Chi Zhang, Matt Carnie, Adam Pockett, Andrew Prescott, Suzanne Thomas, Harrison Ka Hin Lee, Matt Jones 2020. This is the authors' version of the work. It is posted here for your personal use. Not for redistribution. The definitive version was published in CHI 2020, https://doi.org/10.1145/3313831.3376368.

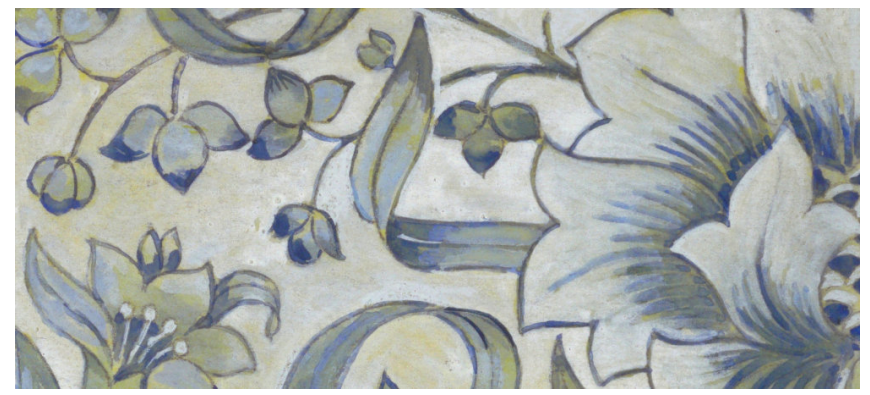

Figure 1. Close-up view of a china tile design by William Morris. Morris' hand-painted decorations feature simple scenes and patterns, often flowers, reduced to stylised elements, and are often painted with a distinctive vibrant blue on a white background.
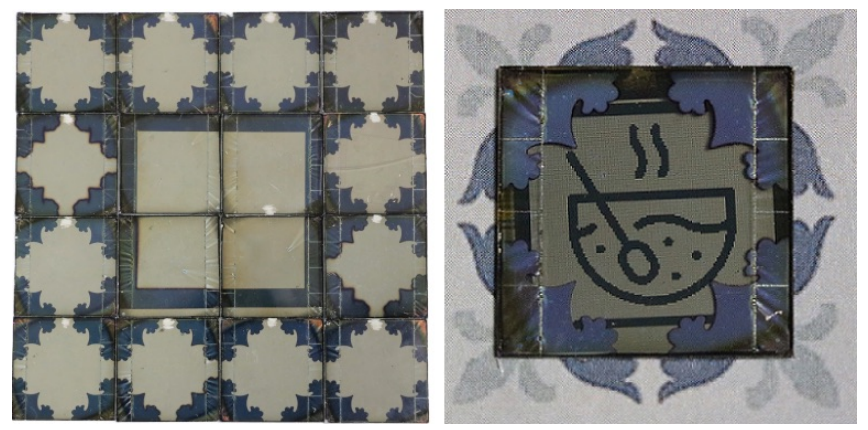

Figure 2. Left: A $4 \times 4$ array of photovoltaic $(P V)$ cells $(12.2 \times 12.2 \mathrm{~cm})$ with transparent windows fabricated in our lab. Right: A PV-Tile with a reflective display and PV surface design matched to its surroundings.

also be advised to extend the rule to ensure that the things created are useful, beautiful and sustainable.

One of the most popular and affordable products produced by Morris' company were painted china tiles (see Fig. 1) that were used to decorate furniture, walls, floors and fireplaces. Such tiles, of course, remain a commonplace form of covering and decoration in many homes globally. While for Morris physical tiles were a ubiquitous material —useful and usable in many locations - for Mark Weiser, father of ubicomp and arguably Morris' counterpart for the digital era, the 'tiles' were computing devices that ranged from pocket-sized tabs to page-sized pads and large screen displays [27].

In our work, inspired by both Morris and Weiser, we are exploring home tiling materials that can deliver elements of Weiser's 
vision and satisfy the extended Morris golden rule. That is, we are working towards tiles that are composed of digital elements—-such as displays, sensors and communication-and photovoltaic materials that can be aesthetically layered on the tile, providing both beauty and the ability to harvest energy to power the digital components. Figure 2 shows a prototype patterned tile, generated after the co-design workshops presented in this paper, composed of a grid of smaller tile segments, and one of these tile segments in close-up.

Unlike previous attempts to use solar-power for sustainable Internet-of-Things (IoT) devices (e.g., [9]), the digital and photovoltaic (PV) materials in our concept are closely coupled: instead of placing a solar panel on the back, above, or some other place separate to the interactive surface, we envisage a semi-transparent PV-Tile sitting directly above the digital display, and for the PV material to be laid down as a pattern instead of the conventional single dark slab. While our initial interactive PV prototype - created in response to the intense participatory workshops presented here-uses rigid materials which are monochrome, advances in material science mean that both flexible and coloured tiling are possible. Figure 3, then, shows example-non-interactive-tiles created in our labs that are patterned and coloured, and a commerciallyavailable flexible PV strip.

With such forms of PV material, allied with digital interactive elements, we envisage a novel class of IoT devices that can be deployed in ways not yet possible with most existing smarthome technologies:

- Firstly, as with conventional tiles that decorate surfaces around the home, they can be more fully embedded in the environment rather than adding additional clutter to a space as separate objects;

- Secondly, as the PV material and the digital elements are closely coupled, changes in the light falling on the tile can be used to directly drive digital interactions: for example, a wave hand-gesture over a table-top tile might change the dining-room lighting ambience; or, the flicker of bathroom candles playing on the tiles around the bath might alter the ebb and flow of a relaxing musical soundtrack; and,

- Finally, as they are intended to be self-powered, they can be built into surfaces and objects without concern for wired infrastructure or the need to open or detach them to replace a depleted battery.

We provide four contributions in this paper, by:

1. Introducing the concept of closely-coupled PV and digital materials to create aesthetically pleasing, useful and sustainable building materials.

2. Presenting a wide range of form-factors, contexts and scenarios surfaced in co-design workshops.

3. Illustrating how shadow-based gestures might enable novel and pleasurable interactions with these materials.

4. Presenting technological designs and initial prototypes to aid others to pursue this new form of material.

To deliver these contributions, we ran two separate co-design workshops with groups of users and experts with backgrounds

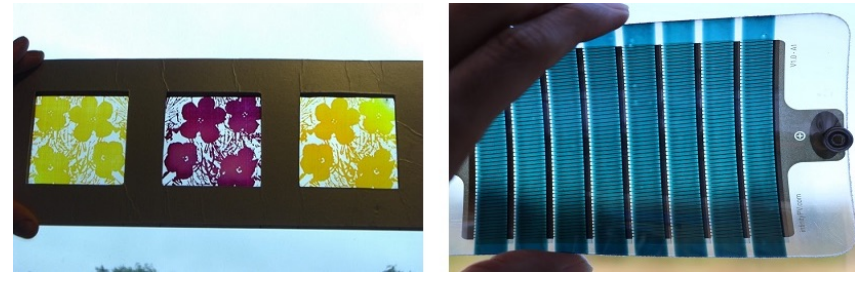

Figure 3. Left: PV cells with a flower pattern and transparent background fabricated in our lab. Right: A commercially available flexible PV strip with semi-transparent background.

including material science, digital interfaces, design and construction. We designed these sessions to enable participants to creatively explore the possibilities afforded by the PV-Tile concept. While this paper focuses on describing the process and results of these workshops, we also provide details of an initial prototype created in response to this work as a starting point for further material science and $\mathrm{HCI}$ innovation.

\section{RELATED WORK}

The PV-Tile concept explored in this paper involves the choreography of energy-harvesting, interaction design and aesthetic considerations. While previous work has addressed these challenges in isolation (e.g., PV for gesture recognition), our work is distinct in that it elaborates the possibilities when all these aspects are considered in harmony. Furthermore, while there have been previous examples that use PV for user input on a limited class of devices, we have uncovered a rich set of possibilities for the PV-Tile concept through co-design.

\section{Exploring concepts through co-design}

Co-design has a long heritage in innovation research and practice. The interfaces, interactions and aesthetic considerations we explore were all driven by using the Dialogue Labs method as described by Lucero et al. [15]. This method has been shown to be effective in taking starting-point conceptual technological propositions and elaborating them. It involves facilitating the generation of ideas by participants by stimulating their creative thinking through a sequence of co-design activities and settings. We were motivated to use the approach by our previous experience of its success in building a design space for another technological concept [8].

\section{Aesthetic design and digital interaction}

In this paper we suggest the value of combining utility, beauty and sustainability. Previous work has also shown how digital interaction can be provided in effective and aesthetically pleasing forms - for example, by embedding computer readable codes in ceramic tableware, wallpaper and the like [21]. Another example involves aesthetics in wireless power transfer with decorative copper coils woven into interactive garments and wearables [28, 29]. Alterwear [7], like our work, considers aesthetics and sustainability. Here, battery-less e-ink displays embedded in clothing, hats and footwear are programmed with visual designs using near-field communication.

\section{Light-based gesture recognition}

A number of prototypes have demonstrated the potential to use light sensing for input. Many of these use technologies 
optimised to detect changes in light (e.g., through photo-diodes (PDs)). GestureLite, then, used a $3 \times 3$-array of low-cost silicon (Si) PDs to detect eight hand gestures with $98 \%$ accuracy in indoor light [10]. LiGest used Si-PDs in ambient light for bodygesture recognition which is agnostic to lighting conditions, position and orientation of the user [26].

In our PV-Tile concept, self-power and light-change detection is the goal. With related aims, Manabe et al. used partial shadowing of $1 \times 6 \mathrm{PV}$ cells for battery-less finger touch and swipe recognition [19]; Li et al. used photo-diodes (PDs) on a watch and a pair of glasses for self-powered finger-based tap and swipe recognition [13]; and, Varshney et al. used solar cells to sense hand gestures in ambient light conditions and combined this with RF communication for ultra-low power battery-free sensing [25]. A recent study has considered the feasibility of using organic solar cells for gesture recognition [16]. This team has further proposed SolarGest, reporting that they were able to detect $96 \%$ of hand gestures in sunlight using transparent solar cells and illustrating the possibility of using the technique on the LCD of a smartwatch [17].

\section{Self-powered digital devices}

Ma et al. survey a number of energy-harvesting and batteryfree devices that have appeared in the market in recent years [18]. These convert properties of light, movement, heat, signals (e.g., Radio Frequency (RF)) etc. to energy. An early example of a simple mechanically-powered push button interface is demonstrated, then, in [23]; while, a display-less battery-free mobile phone that harvests energy from RF signals and from ambient light with tiny photodiodes is described in [24]. More directly related to our work is the IoT device described by Grosse-Puppendahl et al. [9]. This device harvests energy from ambient light using photovoltaic cells at the back of an e-ink display and updates over Bluetooth when enough energy is harvested. A more elaborate sensing and data transmission scheme has been described by Cowell et al. [6]. They show how indoor light energy can be harvested to trickle charge a high-power energy reservoir and also meet the power demand of a wireless sensor network with voltage-triggered sensing and data transmission. In contrast to this prior work, the energy harvesting components of the PV-Tile concept are an integral part of the interface in terms of input capabilities, aesthetic and embedability considerations.

\section{State-of-the-art PV technology}

To achieve the aims of the PV-Tile concept, there needs to be a PV technology that can be highly efficient in low-light conditions (e.g., indoors); that can be fabricated to be transparent or semi-transparent; and, if needed, be patterned and flexible or semi-flexible. For generating power in low light conditions the current state-of-the-art technologies are based on III-IV semiconductors. For example, GaInP cells have been shown to generate $15.6 \mu \mathrm{W} \mathrm{cm} \mathrm{cm}^{-2}$ and $92.6 \mu \mathrm{W} \mathrm{cm}{ }^{-2}$ under $200 \mathrm{~lx}$ and $1000 \mathrm{~lx}$ respectively [20]. Perovskite solar cells are a new type of solar cell manufactured entirely from chemical solutions. Work has recently demonstrated outputs of $19.9 \mu \mathrm{W} \mathrm{cm} \mathrm{c}^{-2}$ and $115.6 \mu \mathrm{W} \mathrm{cm}^{-2}$ under $200 \mathrm{~lx}$ and $1000 \mathrm{~lx}$ respectively [12]. Perovskite cells have other advantages over III-V devices: for example, although both technologies can be made on flexible substrates, only perovskites could be manufactured to be semi-transparent, making them suitable in, say, self-powered interactive window displays, or for integration into wearable technologies. If we consider an opaque perovskite solar cell roughly the size of a credit card $\left(\approx 46 \mathrm{~cm}^{2}\right)$, one would expect to generate 0.9 to $5.3 \mathrm{~mW}$. If semi-transparent devices were desired for aesthetic reasons then one might expect in general less than half of that power. One drawback of the perovskite solar cell is that even as a semi-transparent device the perovskite material retains a brown hue, which may be unappealing for those seeking aesthetically pleasing qualities. As an alternative, organic solar cells (OPV) can offer semi-transparency, flexibility, and a range of colours depending on the type of organic semi-conductor utilised. For these devices one might expect to generate 18 to $45 \mu \mathrm{W} \mathrm{cm}{ }^{-2}$ (200 to $10001 \mathrm{x}$ ) [11] and again it would be expected that semi-transparent devices could generate around half of this.

\section{THE PV-TILE CONCEPT}

The PV-Tile concept marries energy harvesting, interaction and aesthetics via three layers. The PV layer contains the photovoltaic absorber material held within a substrate (e.g., glass or flexible plastic). The material may be laid down in semi-transparent [30] or opaque patterns; and, be coloured [3] or monochromatic. We also envisage un-patterned, semi-transparent PV materials utilising evaporated metal or transparent nanowire electrodes [2] that could be fabricated on this topmost layer, providing designers with further aesthetic possibilities. As well as harvesting energy, the PV material acts as a sensor, providing light changes as inputs to the interactions afforded by the tiles.

The interaction layer contains any displays, actuators or sensors required for the surface. To supplement the light sensors of the PV layer, this layer may contain, for example, ones for proximity and pressure. The control layer contains all of the electronics needed to process signals from the PV and interaction layers and to provide the outputs. A communications module is also needed to allow tiles to interconnect and to communicate with other sources. While for some forms of interface the PV layer may be able to directly harvest enough energy to enable the interfaces in real time, in other cases this base layer is also likely to need a long-life non-replaceable rechargeable battery that is continuously charged when light falls on the PV layer.

The power generated by the PV layer will be much lower than that available to devices connected to mains power or that use a high-capacity battery (such as a mobile phone). With the limited power availability the forms of input and output possible are likely to be different to those on high end digital surfaces in the following respects: the display or audio output quality will be reduced; the ability to sense finegrained touch or shadow gestures will be challenging; and, the responsiveness of the interface might be lower. We suggest that instead of seeing this markedly reduced power availability as an interaction limitation, designers and developers should use the material properties to explore alternative styles of human-device exchange as we demonstrate. 
In terms of sustainability, while the scheme is fully powered by light energy, the PV material will of course have a fixed lifespan after which the tiles would need to be replaced. Commercial PV materials become non-useful after around 25 years, so it is possible to imagine PV-Tiles that will require replacement only very rarely.

\section{Design space}

While each PV-Tile is composed of the three layers, our scheme envisages a diverse range of material forms that provide a rich design space for deployment and interactions:

Shape and size: PV-Tiles can be constructed in a range of dimensions (from postage stamp to wall tile size); and, we are not limited to rectangle form-factors;

Tiling: As in conventional decorative tiles, PV-Tiles are designed to be laid down in groups across surfaces. Different configurations of tiles are possible for aesthetic and interaction design purposes - e.g., multiple contiguous tiles to create a large interactive surface, or the use of spatial distances between tiles or groups of tiles. The tiling can be used to exploit different forms of communication and collaboration between the individual tiles - e.g., all tiles used to display a large image; or, some of the tiles on a surface acting as input elements, others as output.

Flexibility: Some scenarios, objects and contexts that might be envisaged using PV-Tiles require rigid form factors (e.g., in tiling a kitchen); others need to be highly flexible (e.g., when in fabrics), while for others it might be advantageous for the material to be pliant but more sturdy (e.g., when integrated on the cover of a paperback book).

\section{Co-design to elaborate the concept}

Given the conceptual PV-Tile material, as detailed above, we carried out a two-stage co-creation workshop process to explore its potential realisations. We were interested in all aspects of the design space. The first involved members of the general public, while the second leveraged expertise from industrial, public and third-sector stakeholders. The structure of both workshops was based on the dialogue-labs method [15].

\section{WORKSHOP 1 - CO-DESIGN AND END-USERS}

Twenty-one volunteers $(10 \mathrm{M} ; 11 \mathrm{~F})$ aged $18-62$ participated in the workshop along with seven facilitators and observers from the research team. We recruited participants using postings on local community social media and physical posters in locations across the city. Respondents were pre-screened to recruit people who identified as non-tech specialists. After an IRBapproved informed consent process, we ran an ice-breaker drawing game. We then explained that the main purpose of the workshop was to involve the group in the design of digital surfaces with the characteristics of the PV-Tiles concept.

We then gave a 30 minute slide-deck presentation using images and text of: everyday uses of conventional tiles; the concept of coupling PV and digital materials; and, the design parameters. Working prototypes were not demonstrated, as these were constructed after the workshops. We split the volunteers randomly into four groups $(3 \times 5$ and $1 \times 6$ people $)$ and these groups took part in two sessions. The first involved asking participants

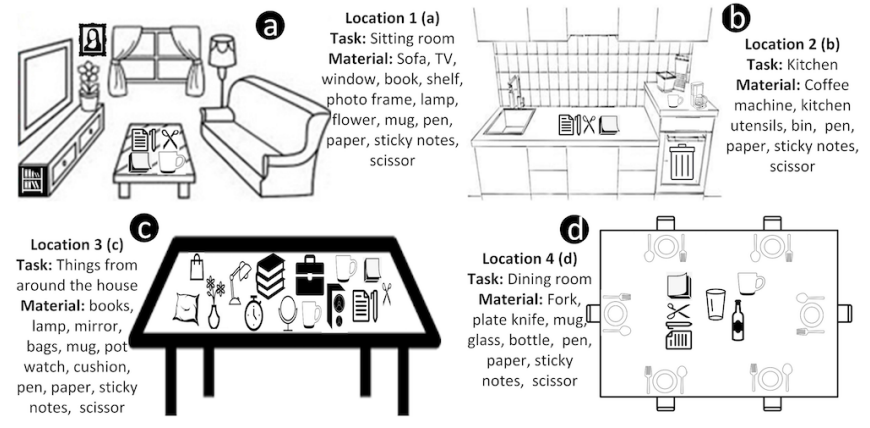

Figure 4. Overview sketch of the dialogue-lab room where Workshop 1 sessions took place. Participants discussed scenarios at four zones: a) sitting room; b) kitchen; c) everyday household objects; d) dining room.

to work to generate example PV-Tile designs, and took place during the first half of the day. After lunch, participants then worked in their groups to consider a form of interaction unique to the PV-Tiles: that is, the use of light-based gestures to control the interfaces.

\section{Session 1: Scenario co-design rounds $(4 \times 30$ mins $)$}

We asked each team to engage with one of four zones that we set up in a large open-plan lab during each 30 minute dialoguelab round. Three zones represented different home-life settings: one was a household sitting room, another a dining room, the third a kitchen. The fourth contained other objects commonly found around a house. In each zone we provided a variety of objects and images to provoke use-case thinking (see Fig. 4).

One facilitator was assigned to each group, and remained with that group as they moved from zone to zone. In addition, three observers moved between groups. The facilitator and observers recorded the ideas and discussion of participants in notebooks and via photos and video. During the $30 \mathrm{~min}$ in each zone, we asked teams to carry out two tasks:

Task 1: Individual (15 mins): With sticky-notes and the objects in the zone as props, the facilitator asked participants to work individually (and in silence) considering where they would place tiles, for what purpose, and how they would interact with them. They then asked each member to demonstrate to the group what they had invented.

Task 2: Group (15 mins): The facilitator asked the team to work together to think about how they might deploy a series of tiles across objects in the zone where the group of tiles would act as a combined interactive surface. After $10 \mathrm{~min}$ of discussion, the group reported back to the facilitator.

\section{Session 2: Shadow play (40 mins)}

After a break for lunch, all participants reconvened in the same groups. We provided each group with two position-adjustable LED lamps for making shadows on a wall or table. While prior work has suggested the use of shadow interactions for projected content [5] and more recently as a control mechanism for objects in the environment [4], this part of the workshop was designed to elicit potential shadow gestures that could be directly detected by the PV-Tiles built into surfaces or objects without other external instrumentation. Each group carried out three tasks, during which we captured photos and notes: 
Task 1: Fun (10 mins): The first task was an ice-breaker where, group-by-group, we asked individual participants to recreate an example shadow they could remember doing as a child and to explain what the shadow meant to them.

Task 2: Shadow competition (15 mins): In this task we asked each group to create as many different shadows as possible, with each participant taking turns in order. A researcher observed each team, and awarded a point for every shadow that was: a) distinct and clear in terms of its shape; b) might communicate something to an interactive surface; and, c) was one that had not been suggested previously during the competition. All groups worked simultaneously, and at the end of the task groups reported back with a winning group (highest number of points) identified.

Task 3: Creating gestures (15 mins): We asked groups to work as a team and create shadow gestures that might instruct a wall of PV-Tiles to carry out the following actions: unlock the home's front door; summon a weather display; mute the music in a sitting room; and send a message of love to another person located elsewhere. We chose these based on common actions mentioned by participants in Session 1. Again, we recorded the discussions, and after each shadow, groups reported back to each other. The best gesture for each action was voted on by all participants as a whole.

\section{Data capture and analysis}

During the workshop, researchers made notes and took photographs and videos. At the end of the workshop these were pooled, and two of the team analysed them to identify themes and categories. These were then discussed and refined with all of the researchers who had been present. We synthesised the most common scenarios and interaction styles into a series of low-fidelity prototypes used as input to the second workshop.

\section{RESULTS OF WORKSHOP 1}

\section{Session 1: Scenario co-design}

Despite the initial briefing by our team about the integration of digital and PV materials, all groups suggested examples of how to use the new surfaces to create novel charging or powering devices that only used the energy harvesting properties of the tiles without any display or interaction element. That is, they took our suggestion that future solar power materials could be made in a variety of shapes, sizes, colours and patterns and with varying degrees of flexibility to propose more aesthetically pleasing embedded charging points. For example, some groups wondered if flexible PV materials and batteries could be woven into a tablecloth that could then be used to charge mobile phones over dinner. Others wondered if a tabletop made up of decorative tiles, patterned with PV material, could be used in the same way.

Turning from these power-generation only use-cases, the other suggestions fell into five categories (with some scenarios falling into more than one category): control devices; notification/information displays; communication/collaboration; user identification; and, artistic/playful surfaces.

Control devices were objects or surfaces which could be touched or gestured at (with the shadow caused by the touch or gesture being sensed by the PV material) to affect change in the environment. So, a place mat on the dinner table was an example of a standalone control device that could be gestured at to change the room's lighting; and, a flexible panel integrated into the arm of a sofa was an example of an embedded control device. The control device would include a display to show the status of the condition being manipulated and to provide feedback as the user carried out a shadow or touch gesture.

Notification/information displays were seen as self-powered surfaces again either placed onto standalone objects (such as a book cover) or built into the environment (e.g., as a set of kitchen tiles). They could provide content received wirelessly from either a user's mobile (e.g., when placing a mobile phone down on a tiled coffee table notifications could be displayed on PV-Tile displays) or directly from a cloud service (e.g., the kitchen tiles showing today's weather forecast). Participants also identified ways that light changes could be used in these scenarios to control the display either explicitly (e.g., hovering over a portion of the display to select an item using the fingers' shadow); or, implicitly (e.g., when a newspaper is placed on the tabletop display, the portion of the display occluded could be sensed and the content reconfigured to remain readable).

Communication/collaboration scenarios emerged in the discussions in the dining room zone. Some of these involved collaborations between group members (e.g., to play simple games; or, to see shadows thrown on one end of the table recreated-for fun - at the other). Others involved people not sitting at the table - e.g., groups suggested ways that the table tiles or objects on the table could be used to view/respond to messages in lieu of looking at their mobile devices, providing more subtle, less rude digital interactions [1].

The user identification scenarios rested on the perceived ability of the PV-Tiles to be able to use the light occlusions created by different users when they touched or held an object. Participants recognised that the ability to widely discriminate between many users might not be possible, but that broad differences in hand sizes between the adult, youth and child members of a household might be identifiable. So, for example, as a person rested their hand on the sitting room coffee table, photos from their own album would be collaged on the tiles.

Artistic/playful scenarios looked at how changes in the light falling on the tile surface might be used to change the display in a pleasing or playful way. For example, in the sitting room zone, one group suggested dynamically mimicking the flicker of candle placed on the table with the PV material sensing the changes to drive the digital display below. Several groups suggested the use of multiple tiles across the dining room table that would change patterns depending on objects moved or placed on the surface, with the surface reacting to the light falling on the surface.

\section{Discussion: PV-Tiles vs. conventional approaches}

The participants were able to see uses of PV-Tiles across a wide range of objects and surfaces. While the sorts of digital augmentation of home settings suggested by participants might be achieved with existing powered or rechargeable conventional technologies, in participants' discussions the following distinct advantages were suggested: 


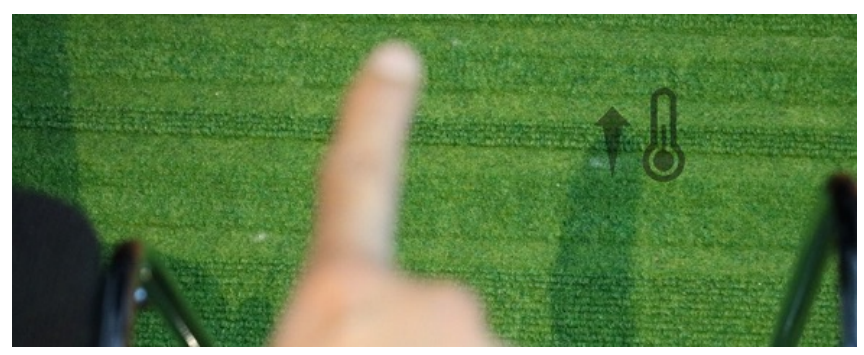

Figure 5. A long-distance shadow cast onto the floor between two sitters at a table gives a way of controlling the room's temperature by activating a floor based PV-Tile without having to reach down to touch it.

- The ability to interact without touching the surface by casting a shadow - this included examples where participants cast a longer shadow for interaction with an object out of reach (see Fig. 5 for an example);

- Flexibility to use explicit (e.g., hand hover gestures) and implicit light interactions (e.g., in the book cover case when a book is open and light falling on it is therefore reduced, a request could be made to retrieve new comments about the book being read);

- The new and pleasurable experience of using light as an interaction medium;

- The non-screen look and feel of the tiles. Participants' comments highlighted their concerns about the increasing dominance of high-resolution touchscreens in their lives and the benefits of being able to augment objects in ways that meant digital materials didn't overpower or dominate the environment; and,

- The lack of a need to plug in a device allowed flexibility in placement and movement and took away what many participants saw as increasing "recharge" burden in their lives where more devices-from phones, to fitness trackers to smart home controllers-need to be monitored for low battery power and recharged regularly.

\section{Low-fidelity prototypes and the dialogue-lab zones}

We made eight low-fidelity prototypes given the participants' discussions and designs to capture the range of surfaces and interactions envisaged across the four dialogue-labs zones. Researchers involved in Workshop 1 created the prototypes in the weeks after that event, using a range of materials including: objects that were used in the first workshop (e.g., plates, cutlery); card and post-it notes; stickers; and, LEDs. Each prototype had a number of states, with elements being manually changed during the interaction. Figure 6 illustrates all eight prototypes as sketches for clarity of presentation in this paper. These prototypes were used as drivers for Workshop 2.

\section{Living room prototypes (Fig. 6, rows 1 and 2)}

Row 1 shows an interactive coffee table made up of a series of PV-Tiles. Tiles in 1a and $1 \mathrm{~b}$ show notification messages from a user's phone. When occluded the messages are moved to still-visible tiles. Image 1c illustrates two other use cases photos are displayed on tiles depending on who touches the table, and the flicker of the candle placed on the table is used to ripple the images on the tiles that can sense these changes.
Row 2 illustrates a flexible PV-Tile built into a fabric armchair. Users can shadow gesture or touch to select to control either the music volume or light intensity via gestures. Once selected the user makes swipe down or up for light controls $(2 \mathrm{a}-\mathrm{c})$.

\section{Dining room prototypes (Fig. 6, row 3)}

Row 3 shows two playful or artistic proposals for the PV materials. In $3 \mathrm{a}$ and $3 \mathrm{~b}$ as the plates and cutlery are placed and removed from the mat, the occluded regions are sensed by the PV-Tile and the patterns reconfigured (note how stars and dots change from $3 a$ to $3 b$ ). In $3 c$ when a glass is placed on the coaster and as the liquid is consumed during the meal, the change in light falling on the tile is sensed (e.g., more light will penetrate the coaster as the more of the drink is consumed) and a self-powered light display reflects these changes.

\section{Kitchen prototypes (Fig. 6, row 4)}

Image 4a shows a set of five PV-Tiles displaying a recipe to the user (in the prototype represented via sticky notes). Using an upwards or downwards shadow gesture on the wall allows the user to scroll to the next or previous page without having to touch the surface. By carrying out a swipe right shadow gesture across the wall (4b), the tiles display the weather forecast (again represented as a series of sticky notes).

\section{Everyday object prototypes (Fig. 6, row 5)}

Image 5a shows a semi-rigid PV-Tile built into a book cover showing the page last read on the e-book version of the physical book plus reviews and comments from other readers. Images $5 b$ and $5 c$ are flexible tiles encapsulated around a coffee mug showing notifications. By sensing the light changes as the mug is rotated, new notification cards are displayed. Image $5 \mathrm{~d}$ is a simple plant pot moisture level notifier incorporating an e-ink display and moisture sensor (the face on the display showing healthy—smiley—or unhealthy—frown—levels).

\section{Session 2: Shadow play}

Task 1 - Fun

There was a great deal of laughter from participants as they took it in turns to make shadows, and positive appreciation by onlookers in the rest of each group. A wide range of shadows from "rabbits" to "crocodiles" were created, with some participants also suggesting interaction meanings for their gesture (e.g., a bird shape to send a message).

\section{Task 2 - Shadow competition}

128 gestures were created by the teams. The purposes intended fell into one of three main categories: $28 \%$ to control aspects of media playback (e.g., turn TV on/off; increase/decrease volume, etc.); $31 \%$ to control physical elements in the environment (e.g., unlock a door elsewhere in the house; turn off lights); and, $41 \%$ to initiate some form of communication (e.g., start a call; or, send a message). We noted a wide range of alternative gestures for similar actions between the groups.

While we did not specify how participants made their gestures, most created them using either one or two hands; however, in a few cases, other parts of their body (head, arms and torsos) were involved. Most of the gestures created involved dynamics; that is, movements of the shadows (e.g., swiping, clapping 


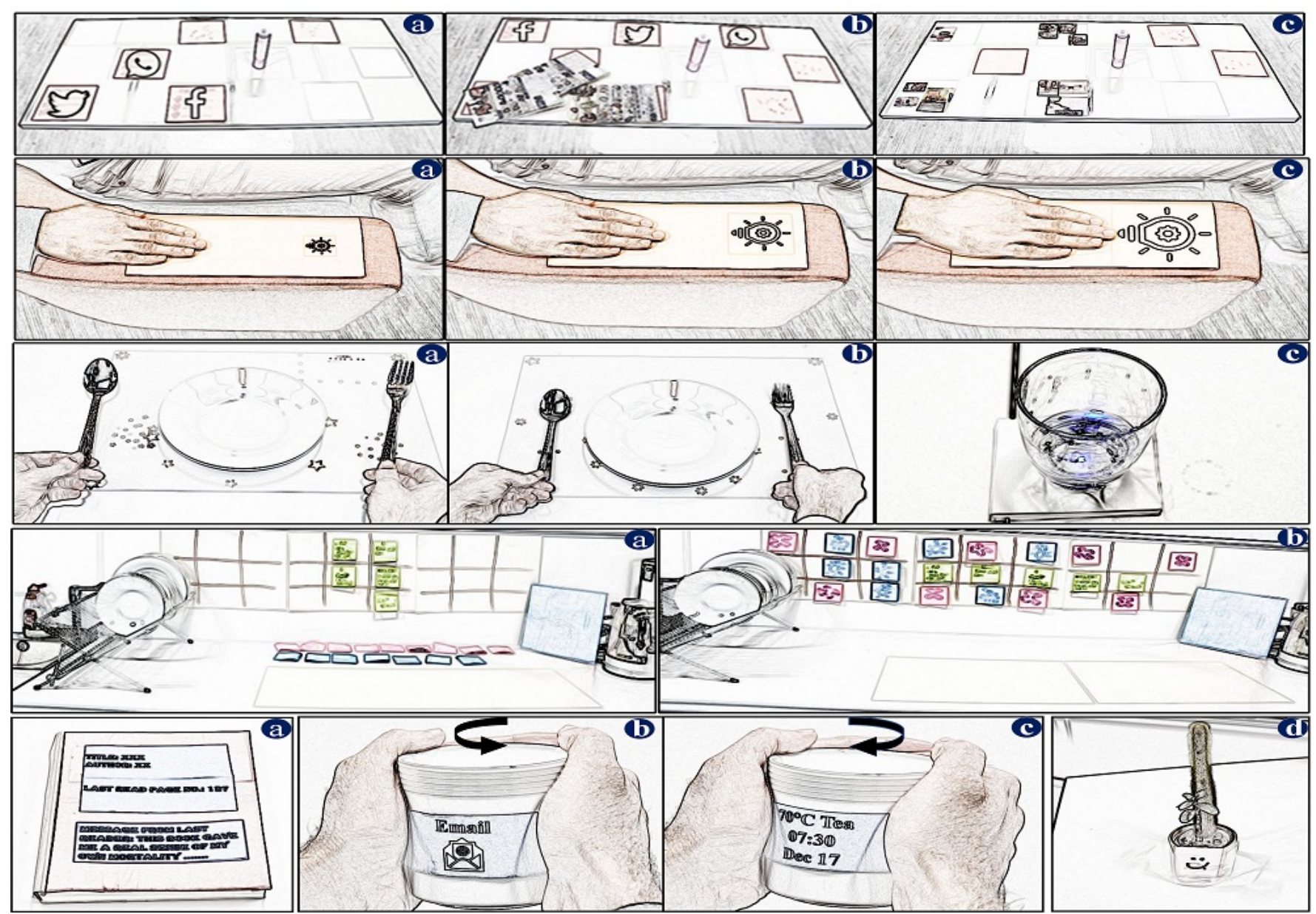

Figure 6. Illustrative sketches of the low-fidelity PV-Tile prototypes inspired by Workshop 1 and used in Workshop 2. Living room: interactive coffee table (row 1, images a-c) and remote control (row 2, a-c). Dining room: playful table place mat (row 3, a-b) and coaster (row 3, c). Kitchen: interactive wall surface (row 4, a-b). Everyday objects: book cover (row 5, a), notification mug (row 5, b-c) and flower pot (row 5, d).

hands, turning a finger). $77 \%$ of gestures involved small movements (e.g., opening and folding of hands); and, $23 \%$ used larger movements (e.g., waving hands back and forth).

\section{Task 3 - Creating gestures}

In designing shadow gestures for the set tasks, again we saw a range of suggestions including ones that were figurative and others that were abstract - for example, to mute the music in a room one group made a shadow representing a music conductor's movements as they silenced an orchestra, while another created a pinch gesture with the thumb and first finger closing together to express silence. One group thought it would be worth considering enabling users to use sign language handshapes as the basis of some of the human-surface interactions.

The gestures that were seen as the best by the participants for each of our imagined shadow-to-device interactions were:

Unlock a door: A one-handed rotation as if holding a key

Display weather: a sun-like shape with rays using two hands Mute music: a pinch gesture using one hand

Send a message of love: making the shape of a heart using two hands

\section{Discussion: Shadow play}

Throughout this session there was a strong sense of fun and enjoyment. As we saw in the morning session, participants appreciated the novelty and potential pleasure in interacting with surfaces through shadow gestures. Participants were highly creative and individual in designing gestures, suggesting any real system should be able to allow a user to personalise the gestures they associate with different interactions.

In this and the morning scenario session we did not ask participants to consider practical issues of shadows and their recognition. That is, we did not explore with them how their interactions might be distinguished from shadows cast by other movements in the environment or changes in the ambient light falling on a surface (as, for example, when a cloud passes across the sun). In this session, though, we noted that the dynamics used in most of the gestures might aid a real system implementation as a short distinct movement (e.g., hand clapping) might be easier to discriminate than a static one.

\section{WORKSHOP 2 - CO-DESIGN AND EXPERTS}

Nineteen experts (13M; 6F) aged 26-62 participated in this second workshop carried out a month after Workshop 1. We 
recruited participants to include academics with interests in material science, design and computing; and, non-academics (from private and public companies) involved in either sectors that might find the PV-Tile materials of use for services they provided (e.g., one participant was a member of major broadcasting organisation; others were from health providers, etc.), or that might use the materials directly in their work (e.g., building firms). We asked each participant to self-classify their expertise across six pre-defined categories: design (8 participants), materials science (7), health and well-being (3), construction (3), manufacturing (4) and computing/IT (10). ${ }^{1}$

In addition, six researchers (four design/computing, two materials science/manufacturing) acted as observers and facilitators. After presenting the introductory slide deck from Workshop 1 , with additional technical, architectural and design details, we split the participants into three groups $(2 \times 6$ and $1 \times 7$ people). We formed groups so that each consisted of a mix of backgrounds and interests. These then took part in two tasks:

Task 1: Initial reactions A group discussion on first impressions of the concepts and technologies $(30 \mathrm{~min})$ followed by feedback to all participants.

Task 2: Output discussion and reflections A zone-by-zone discussion of outputs and low-fidelity prototypes generated from the first workshop. Groups spent $45 \mathrm{~min}$ in each zone. During each session, a facilitator explained the zone and outcomes provided by the previous participants. We then asked the experts to comment on and extend the ideas from their interaction design, application and technical perspectives.

\section{RESULTS OF WORKSHOP 2}

\section{Task 1: Initial reactions}

The consensus was that the proposed materials had a high degree of potential in terms of providing new forms of useful and aesthetically pleasing construction material that could be built into homes, public settings and workplaces.

While there was a strong agreement that the combination of self-power and digital interactions was timely and a good step towards sustainable innovation, there was an equally clear challenge from the group regarding potential negative unintended consequences. Firstly, participants pointed out that this new material could lead to even more digital devices that would consume resources apart from the energy to drive them. Secondly, while building materials (such as tiles on walls) are often left in place for many years, if the digital interactions or services afforded by our new materials become obsolete, this might actually encourage people to "upgrade" them much more often then they currently do. Groups noted that many tiles in homes are put in place by Do-It-Yourself enthusiasts, often the homeowner. They challenged our team to think about how these non-experts could not only install the tiles but could also configure their use and display content without becoming programming or interaction design experts.

\section{Task 2: Output discussion and reflections}

From the zone-by-zone discussions of the low-fidelity prototypes, participants provided suggestions and comments that

\footnotetext{
${ }^{1}$ Note: several participants identified expertise in more than one area.
}

surfaced practical challenges, extended the earlier ideas, and endorsed the value of non-expert participant use-cases:

Practical considerations: Material science experts commented on the difficulties of ensuring that objects augmented with PV-Tiles could withstand any exposure to water (e.g., by washing) or heat (e.g., placing a hot mug of coffee on the surface). They also noted the health and safety aspects inherent in PV materials. In commercial versions of such tiles, such considerations might be addressed through robust encapsulation.

A recurrent discussion across all the zones was the very lowlevels of power availability that interfaces and interactions would have to accommodate. Suggestions to address this included power-saving designs (e.g., requiring a user to explicitly activate the surface by a touch gesture); and, using this constraint to stimulate a new form of smart home interaction philosophy that aims at "enough resolution" (of display or gesture) to be useful and usable.

Extensions: The user-identification opportunities presented in the earlier workshop were extended by the expert group, who suggested users could create personalised shadow signatures (for instance, the sorts of fun childhood shadows demonstrated by participants in Workshop 1). The communication and collaboration scenarios from the dining room zone were elaborated to consider restaurant settings: e.g., using the table-top tiles to display and pay for the bill via an NFC reader for card transactions; and, for summoning a table server.

Endorsements: Participants appreciated the utility and novelty of non-touch shadow gestures. For example, they commented on the value of not having to touch the recipe display in the kitchen scenario when hands are dirty or when using utensils. As with non-experts, this group saw new opportunities and advantages coming from the material form factor and performance of PV-Tiles. That is, they contrasted the interfaces and interactions possible against those provided via current ubiquitous high-resolution, highly featured touch.

\section{PV-TILE PROTOTYPE}

Following the co-design workshops, we built a working PVTile prototype to explore the viability of self-powered PVbased interaction (see Fig. 7, left). The workshops identified a range of rigid, semi-flexible and fully-flexible deployments, along with a diverse set of sensors, actuators and displays. For this first prototype, we focus on a rigid form-factor for two of the scenarios identified in the workshops.

We did not evaluate the hardware demonstrators with participants, as their purpose was to surface technical viability and limitations. The validity of the applications they embody, however, is supported by their co-design in Workshop 1 and the use and endorsement of low-fidelity versions in Workshop 2.

\section{Prototype components}

We created the prototype using the following implementation choices for the three layers in the conceptual PV-Tile model:

PV layer: Organic photovoltaic (OPV) cells

The OPV cells serve a dual function: they both harvest energy from ambient lighting; and, detect shadows for gesture 

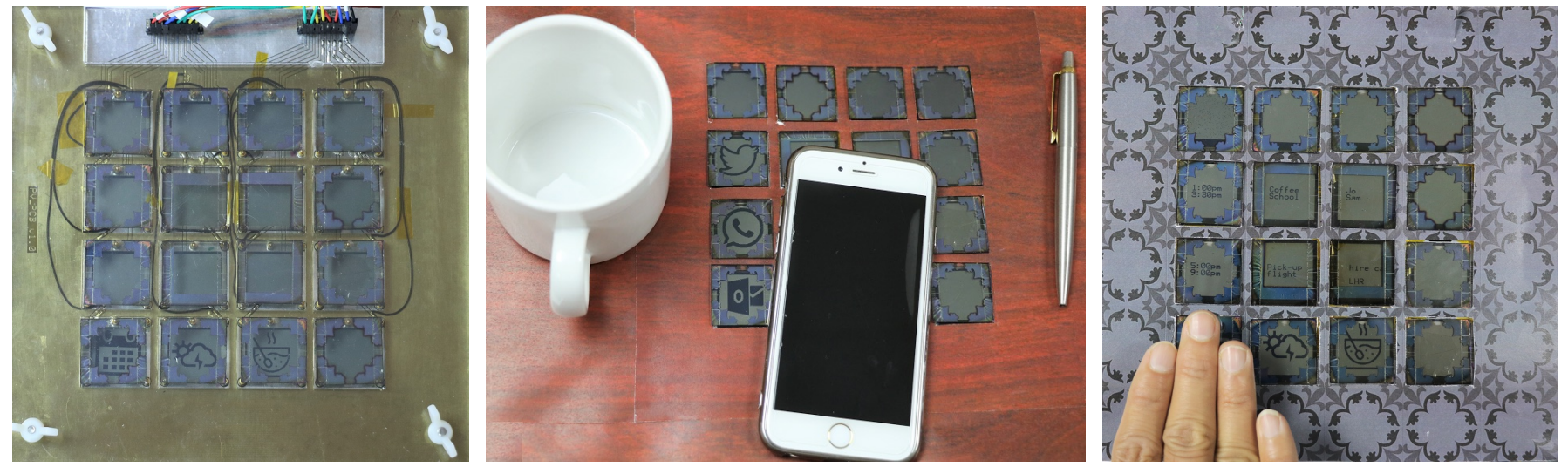

Figure 7. Left: our demonstrator PV-Tile prototype. Sixteen OPV cells are organised into a $4 \times 4$ array. Digital displays are visible beneath the tiles (see icons in bottom row). Other images show the PV-Tile in simulated interaction contexts. Centre: a tabletop scenario, with displayed elements moving when occluded (see Fig. 6, row 1, images a and b). Right: a kitchen scenario, using shadow gestures to select and scroll through content (Fig. 6, row 4).

\begin{tabular}{|c|c|c|}
\hline $\mathrm{MoO}_{3} / \mathrm{Ag}$ & & $\mathrm{MoO}_{3} / \mathrm{Ag}$ \\
\hline \multicolumn{3}{|c|}{ PCE-10:PC ${ }_{71} \mathrm{BM}$} \\
\hline \multicolumn{3}{|c|}{ Zinc Oxide } \\
\hline \multicolumn{3}{|c|}{ Indium Tin Oxide } \\
\hline & Glass substrate & \\
\hline
\end{tabular}

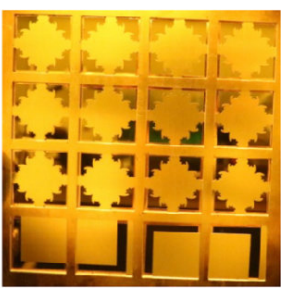

Figure 8. The cross section of an organic PV cell (left). The top of each cell consists of a silver metal pattern created by a shadow mask (right).

sensing. We fabricated sixteen cells (size $28 \times 28 \mathrm{~mm}$ ) in a cleanroom using a relatively simple process as demonstrated in the accompanying video figure. The PV cell is a layered structure as shown in Fig. 8 (left), fabricated on top of a commercial indium tin oxide (ITO) coated $(15 \Omega / \mathrm{sq})$ glass substrate bottom-up one layer at a time.

The decorative pattern is laid down as the top silver metal layer. To achieve this, in the final step we deposited $10 \mathrm{~nm}$ of $\mathrm{MoO}_{3}$ and $130 \mathrm{~nm}$ of $\mathrm{Ag}$ by evaporation (without breaking the vacuum between layers) through a shadow pattern mask as shown in Fig. 8 (right), which represents the negative of the pattern. The electrical contacts to the ITO and silver layers of the tile are achieved using spring-loaded contacts.

The sixteen OPV cells are organised into a $4 \times 4$ array (see Fig. 7, left). Within the grid we used a low power $(\approx 3 \mu \mathrm{W}$ at $3.3 \mathrm{~V}$ in standby mode) passive infrared (PIR) sensing module as a motion detector to signal to the bottom layer electronics when to switch between energy harvesting mode and gesture sensing mode. That is, when there is no user interaction the tiles harvest energy, and when movements near the tile are sensed, the gesture sensing algorithms are activated.

\section{Interaction layer}

Low power polymer network liquid crystal (PNLC) displays ${ }^{2}$ serve as the information display elements of the tile. Each display is of a similar size to the OPV cells in the top level. Sixteen displays are placed directly underneath the cell array.

\footnotetext{
${ }^{2}$ LS013B7DH03; ${ }^{3}$ MSPEXP-430F5529; ${ }^{4}$ BOOSTXL-SHARP128; ${ }^{5}$ STEVAL-ISV021V1.
}

\section{Control layer}

This level contains the ultra low power electronics and energy storage devices needed make the interface function as required. At its core is a microcontroller ${ }^{5}$ that runs the algorithms used to detect gestures and sends images and text to the displays via a driver module. ${ }^{5}$ Custom electronic switches allow energy from the OPV cells to be routed to an energy harvester module ${ }^{5}$, which charges a small lithium-ion battery when the interface is not being used to detect gestures.

\section{Hardware performance}

We evaluated the PV-Tile's performance under indoor lighting. Each cell typically produced about $12 \mu \mathrm{W}$ per $100 \mathrm{~mm}^{2}$ under laboratory fluorescent light $(\approx 8401 \mathrm{~lx})$ with $300 \mathrm{mV}$ opencircuit voltage and $40 \mu \mathrm{A}$ short-circuit current. The patterned silver layer reduced the energy harvesting capability of the PV cells. However, the reduction was not proportional to the reduction in area of the metal contact. Further optimisation in depositing the silver layer with the shadow mask or fabricating a transparent/semi-transparent layer over the silver layer could increase the energy harvesting performance of the cells.

The prototype is designed for low energy consumption during both energy harvesting and interactive modes. During energy harvesting, the microcontroller is placed into its lowest power mode, where its current consumption is $\approx 1.2 \mu \mathrm{A}$ at $3.3 \mathrm{~V}$. The PIR sensor consumes $1 \mu \mathrm{A}$ during energy harvesting and $<100 \mu \mathrm{A}$ when activated. The PNLC digital displays require $10 \mu \mathrm{W}$ per refresh cycle. Overall, the current consumption of the prototype is $\approx 8.2 \mu \mathrm{A}$ in energy harvesting mode and $\approx 5 \mathrm{~mA}$ in active mode (i.e., when gestures are being detected). Using these figures, we estimate that with sixteen OPV cells producing a total of $775 \mu \mathrm{W}$ under $840 \mathrm{~lx}$ fluorescent lighting, the prototype can detect approximately one gesture every five minutes for energy-neutral operation.

\section{Gesture recognition}

When the PIR sensor detects movement near the device, it is automatically switched to interaction mode. To demonstrate simple scenarios, we implemented two modes of PV-based gesture sensing: hover-input with partial tile shadowing; and, 


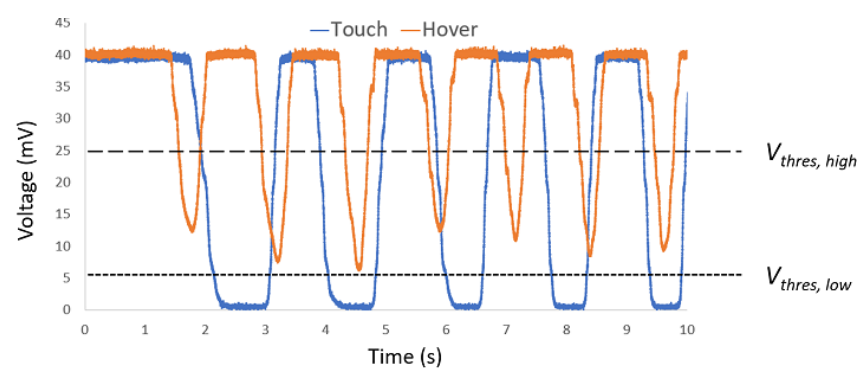

Figure 9. PV-Tile prototype voltages for seven hover (i.e., hand above, but not touching) and five touch (i.e., contact) inputs. Hover and touch are detected when between threshold voltages and below the low threshold voltage, respectively.

touch-input with full occlusion of the PV area. Figure 9 illustrates the change in voltage that occurs during a series of gesture interactions. When the voltage drops below the lower threshold the system assumes a touch gesture has occurred. If the voltage is below the upper threshold but above the lower, the system actions a hover gesture. As each of the tiles can independently sense hover gestures, by monitoring dynamic changes across the full prototype we are able to implement mid-air "swipes": i.e., a hover gesture moving left, right, up or down across the prototype. We have included the gesture recognition implementation in the accompanying PV-Tile toolkit. ${ }^{6}$

\section{Demonstrators}

We used the PV-Tile prototype to implement design ideas created in the workshops (see Fig. 7, centre and right). In the tabletop demonstrator, users receive visual notifications (e.g., Twitter, WhatsApp, email). When an object occludes part of the tile, the display is reconfigured so that notifications are still visible. In the kitchen wall demonstrator, users can select calendar, weather and recipe apps by hovering above the icons, and can scroll or clear the digital content by swiping over the content. Please refer to the video figure accompanying this paper to see the demonstrators in action.

\section{CONCLUSIONS AND FUTURE WORK}

This work took as its starting point a desire to explore a new class of sustainable IoT technologies. While previous research has demonstrated the use of PV materials to power such devices and detect gestures for interaction, we have introduced a concept_PV-Tiles-that combines these abilities and, in addition, supports aesthetic and embedability considerations.

The participants in our co-design workshops populated a design space with a broad range of form-factors, interaction techniques and uses for the concept, demonstrating the potential for such materials to provide useful, sustainable and beautiful interactive surfaces that are infrastructure-free.

While we have also demonstrated the viability of the concept with a first prototype that could enable self-powered interfaces embedded, for instance, in walls or tabletops, there is much work to do to enable the full range of possibilities uncovered through the co-design sessions; and, as the expert workshop participants noted, to enable real-world deployments. This

\footnotetext{
${ }^{6}$ See: https://deliot.me/toolkit.html
}

includes using and driving advances in material science to provide for flexible and semi-flexible tiles; the addition of sophisticated patterns and colouring; and, optimisation of the power harvesting properties of the tiles and energy-neutral design of the gesture recognition algorithms.

From a broader interaction design perspective, the work has surfaced new opportunities. Today's interactive devices are increasingly high-resolution in terms of both user input (e.g., [14]), output (e.g., the very high specification of the displays on the latest foldable devices), and high-capacity wireless networking. All of this comes at high cost in terms of both energy consumption and the re-charge burden that was reported by participants in the first workshop. Our work suggests attention should be paid to a "just-enough" style of interface: just enough input; output; data; updates, and so on. The benefits of this approach as appreciated by workshop participants may be two-fold: the reduction in both energy consumption and the need for users to keep tending to their devices by either replacing batteries or recharging; and, as a way of enabling useful digital interactions that are less all-consuming and demanding. The co-design participants presented ideas (e.g., the artistic or playful scenarios) and interactions (e.g., the almost super-power of casting a long shadow to interact with a surface out-of reach) that indicate limitations brought on by sustainability and embedability considerations do not mean that such surfaces cannot be powerful and pleasurable.

As noted in the expert workshop, there is also interesting future work to be done on ensuring materials like PV-Tiles can be used in a DIY context. We plan now to explore how non-specialists can create aesthetic designs and configure tile behaviours just as they can choose how to physically arrange today's non-digital tiles.

\section{ACKNOWLEDGEMENTS}

This work was funded EPSRC (EP/R032750/1). We gratefully acknowledge the comments provided by reviewers on earlier drafts, and Ben Jones for input on potential prototype gestures.

\section{REFERENCES}

[1] Fraser Anderson, Tovi Grossman, Daniel Wigdor, and George Fitzmaurice. 2015. Supporting Subtlety with Deceptive Devices and Illusory Interactions. In Proceedings of the 33rd Annual ACM Conference on Human Factors in Computing Systems (CHI'15). ACM, New York, NY, USA, 1489-1498. DOI : https://doi.org/10.1145/2702123.2702336

[2] Colin D. Bailie, M. Greyson Christoforo, Jonathan P. Mailoa, Andrea R. Bowring, Eva L. Unger, William H. Nguyen, Julian Burschka, Norman Pellet, Jungwoo Z. Lee, Michael Grätzel, Rommel Noufi, Tonio Buonassisi, Alberto Salleo, and Michael D. McGehee. 2015. Semi-transparent perovskite solar cells for tandems with silicon and CIGS. Energy Environ. Sci. 8 (2015), 956-963. Issue 3. DOI : https://doi .org/10.1039/C4EE03322A

[3] Yi-Hong Chen, Chang-Wen Chen, Zheng-Yu Huang, Wei-Chieh Lin, Li-Yen Lin, Francis Lin, Ken-Tsung 
Wong, and Hao-Wu Lin. 2014. Microcavity-Embedded, Colour-Tuneable, Transparent Organic Solar Cells. Advanced Materials 26, 7 (2014), 1129-1134. DOI: https://doi.org/10.1002/adma.201304658

[4] Eria Chita, Yuta Sugiura, Sunao Hashimoto, Kai Kunze, Masahiko Inami, and Masa Ogata. 2015. Silhouette Interactions: Using the Hand Shadow As Interaction Modality. In Adjunct Proceedings of the 2015 ACM International Joint Conference on Pervasive and Ubiquitous Computing and Proceedings of the 2015 ACM International Symposium on Wearable Computers (UbiComp/ISWC'15 Adjunct). ACM, New York, NY, USA, 69-72. DOI : https://doi.org/10.1145/2800835.2800870

[5] Lisa G. Cowan and Kevin A. Li. 2011. ShadowPuppets: Supporting Collocated Interaction with Mobile Projector Phones Using Hand Shadows. In Proceedings of the SIGCHI Conference on Human Factors in Computing Systems (CHI '11). ACM, New York, NY, USA, 2707-2716. DOI :

https://doi.org/10.1145/1978942.1979340

[6] M. A. Cowell, B. P. Lechene, P. Raffone, J. W. Evans, A. C. Arias, and P. K. Wright. 2016. Wireless sensor node demonstrating indoor-light energy harvesting and voltage-triggered duty cycling. Journal of Physics: Conference Series 773 (nov 2016), 012033. DOI: https://doi .org/10.1088/1742-6596/773/1/012033

[7] Christine Dierk, Molly Jane Pearce Nicholas, and Eric Paulos. 2018. AlterWear: Battery-Free Wearable Displays for Opportunistic Interactions. In Proceedings of the 2018 CHI Conference on Human Factors in Computing Systems (CHI '18). ACM, New York, NY, USA, Article 220, 11 pages. DOI :

https://doi.org/10.1145/3173574.3173794

[8] Alix Goguey, Cameron Steer, Andrés Lucero, Laurence Nigay, Deepak Ranjan Sahoo, Céline Coutrix, Anne Roudaut, Sriram Subramanian, Yutaka Tokuda, Timothy Neate, Jennifer Pearson, Simon Robinson, and Matt Jones. 2019. PickCells: A Physically Reconfigurable Cell-composed Touchscreen. In Proceedings of the 2019 CHI Conference on Human Factors in Computing Systems (CHI '19). ACM, New York, NY, USA, Article 273, 14 pages. DOI : https://doi.org/10.1145/3290605.3300503

[9] Tobias Grosse-Puppendahl, Steve Hodges, Nicholas Chen, John Helmes, Stuart Taylor, James Scott, Josh Fromm, and David Sweeney. 2016. Exploring the Design Space for Energy-Harvesting Situated Displays. In Proceedings of the 29th Annual Symposium on User Interface Software and Technology (UIST '16). ACM, New York, NY, USA, 41-48. DOI:

https://doi.org/10.1145/2984511.2984513

[10] Mahina-Diana Kaholokula. 2016. Reusing Ambient Light to Recognize Hand Gestures (Technical Report TR2016-797). Dartmouth College Computer Science, $1-17$.
[11] Harrison K.H. Lee, Zhe Li, James R. Durrant, and Wing C. Tsoi. 2016. Is organic photovoltaics promising for indoor applications? Applied Physics Letters 108, 253301 (2016). DOI :

https://doi.org/10.1063/1.4954268

[12] Harrison Ka Hin Lee, Jérémy Barbé, Simone M P Meroni, Tian Du, Chieh-Ting Lin, Adam Pockett, Joel Troughton, Sagar M Jain, Francesca De Rossi, Jennifer Baker, Matthew J Carnie, Martyn A McLachlan, Trystan M Watson, James R Durrant, and Wing C Tsoi. 2019. Outstanding Indoor Performance of Perovskite Photovoltaic Cells - Effect of Device Architectures and Interlayers. Solar RRL 3, 1 (2019), 1800207. DOI : https://doi.org/10.1002/solr.201800207

[13] Yichen Li, Tianxing Li, Ruchir A. Patel, Xing-Dong Yang, and Xia Zhou. 2018. Self-Powered Gesture Recognition with Ambient Light. In Proceedings of the 31st Annual ACM Symposium on User Interface Software and Technology (UIST '18). ACM, New York, NY, USA, 595-608. DOI:

https://doi.org/10.1145/3242587.3242635

[14] Jaime Lien, Nicholas Gillian, M. Emre Karagozler, Patrick Amihood, Carsten Schwesig, Erik Olson, Hakim Raja, and Ivan Poupyrev. 2016. Soli: Ubiquitous Gesture Sensing with Millimeter Wave Radar. ACM Trans. Graph. 35, 4, Article 142 (July 2016), 19 pages. DOI :

https://doi.org/10.1145/2897824.2925953

[15] Andrés Lucero, Kirsikka Vaajakallio, and Peter Dalsgaard. 2012. The dialogue-labs method: process, space and materials as structuring elements to spark dialogue in co-design events. CoDesign 8, 1 (2012), 1-23. DOI :

https://doi.org/10.1080/15710882.2011.609888

[16] Dong Ma, Guohao Lan, Mahbub Hassan, Wen Hu, Mushfika Baishakhi Upama, Ashraf Uddin, and Moustafa Youssef. 2018. Gesture Recognition with Transparent Solar Cells: A Feasibility Study. In Proceedings of the 12th International Workshop on Wireless Network Testbeds, Experimental Evaluation $\mathcal{E}$ Characterization (WiNTECH '18). ACM, New York, NY, USA, 79-88. DOI : https://doi .org/10.1145/3267204.3267209

[17] Dong Ma, Guohao Lan, Mahbub Hassan, Wen Hu, Mushfika B. Upama, Ashraf Uddin, and Moustafa Youssef. 2019. SolarGest: Ubiquitous and Battery-free Gesture Recognition Using Solar Cells. In The 25th Annual International Conference on Mobile Computing and Networking (MobiCom '19). ACM, New York, NY, USA, Article 12, 15 pages. DOI: https://doi.org/10.1145/3300061.3300129

[18] Dong Hua Ma, Guohao Lan, Mahbub Hassan, Wen Hu, and Sajal K. Das. 2019. Optimizing Sensing, Computing, and Communication for Energy Harvesting IoTs: A Survey. CoRR abs/1905.03949 (2019). 
[19] Hiroyuki Manabe and Masaaki Fukumoto. 2012. Touch Sensing by Partial Shadowing of PV Module. In Adjunct Proceedings of the 25th Annual ACM Symposium on User Interface Software and Technology (UIST Adjunct Proceedings '12). ACM, New York, NY, USA, 7-8. DOI :

https://doi.org/10.1145/2380296.2380301

[20] I. Mathews, P. J. King, F. Stafford, and R. Frizzell. 2016. Performance of III-V Solar Cells as Indoor Light Energy Harvesters. IEEE Journal of Photovoltaics 6, 1 (Jan 2016), 230-235. DOI :

https://doi.org/10.1109/JPHOTOV.2015.2487825

[21] Rupert Meese, Shakir Ali, Emily-Clare Thorne, Steve D. Benford, Anthony Quinn, Richard Mortier, Boriana N. Koleva, Tony Pridmore, and Sharon L. Baurley. 2013. From Codes to Patterns: Designing Interactive Decoration for Tableware. In Proceedings of the SIGCHI Conference on Human Factors in Computing Systems (CHI '13). Association for Computing Machinery, New York, NY, USA, 931-940. DOI: https://doi.org/10.1145/2470654.2466119

[22] William Morris. 1882. Hopes and Fears for Art. Ellis \& White.

[23] Joseph A. Paradiso and Mark Feldmeier. 2001. A Compact, Wireless, Self-Powered Pushbutton Controller. In Proceedings of the 3rd International Conference on Ubiquitous Computing (UbiComp '01). Springer-Verlag, Berlin, Heidelberg, 299-304.

[24] Vamsi Talla, Bryce Kellogg, Shyamnath Gollakota, and Joshua R. Smith. 2017. Battery-Free Cellphone. Proc. ACM Interact. Mob. Wearable Ubiquitous Technol. 1, 2, Article 25 (June 2017), 20 pages. DOI : https://doi.org/10.1145/3090090

[25] Ambuj Varshney, Andreas Soleiman, Luca Mottola, and Thiemo Voigt. 2017. Battery-free Visible Light
Sensing. In Proceedings of the 4th ACM Workshop on Visible Light Communication Systems (VLCS '17).

ACM, New York, NY, USA, 3-8. DOI :

https://doi.org/10.1145/3129881.3129890

[26] Raghav H. Venkatnarayan and Muhammad Shahzad. 2018. Gesture Recognition Using Ambient Light. Proc. ACM Interact. Mob. Wearable Ubiquitous Technol. 2, 1, Article 40 (March 2018), 28 pages. DOI :

https://doi .org/10.1145/3191772

[27] Mark Weiser. 1999. The Computer for the 21st Century. SIGMOBILE Mob. Comput. Commun. Rev. 3, 3 (July 1999), 3-11. DOI :

https://doi.org/10.1145/329124.329126

[28] P. Worgan and M. Fraser. 2016. Garment level power distribution for wearables using inductive power transfer. In 2016 9th International Conference on Human System Interactions (HSI). IEEE Computer Society, Washington, DC, USA, 277-283. DOI : https://doi.org/10.1109/HSI. 2016.7529644

[29] Paul Worgan, Odysseas Pappas, Themis Omirou, and Michael Collett. 2015. Flexible On-body Coils for Inductive Power Transfer to IoT Garments and Wearables. In Proceedings of the 2015 IEEE 2nd World Forum on Internet of Things (WF-IoT) (WF-IOT'15). IEEE Computer Society, Washington, DC, USA, 297-298. DOI :

https://doi .org/10.1109/WF-IoT . 2015.7389069

[30] Ligang Yuan, Zhaowei Wang, Ruomeng Duan, Peng Huang, Kaicheng Zhang, Qiaoyun Chen, Nageh K. Allam, Yi Zhou, Bo Song, and Yongfang Li. 2018. Semi-transparent perovskite solar cells: unveiling the trade-off between transparency and efficiency. J. Mater. Chem. A 6 (2018), 19696-19702. Issue 40. DOI :

https://doi.org/10.1039/C8TA07318] 